Tohoku Math. J.

66 (2014), 523-537

\title{
PERTURBATION OF DIRICHLET FORMS AND STABILITY OF FUNDAMENTAL SOLUTIONS
}

\author{
MASAKI WADA \\ (Received September 25, 2013, revised October 28, 2013)
}

\begin{abstract}
Let $\left\{X_{t}\right\}_{t \geq 0}$ be the $\alpha$-stable-like or relativistic $\alpha$-stable-like process on $\boldsymbol{R}^{d}$ generated by a certain symmetric jump-type regular Dirichlet form $(\mathcal{E}, \mathcal{F})$. It is known in [5, 7] that the transition probability density $p(t, x, y)$ of $\left\{X_{t}\right\}_{t>0}$ admits the two-sided estimates. Let $\mu$ be a positive smooth Radon measure in a certain class and consider the perturbed form $\mathcal{E}^{\mu}(u, u)=\mathcal{E}(u, u)-(u, u)_{\mu}$. Denote by $p^{\mu}(t, x, y)$ the fundamental solution associated with $\mathcal{E}^{\mu}$. In this paper, we establish a necessary and sufficient condition on $\mu$ for $p^{\mu}(t, x, y)$ having the same two-sided estimates as $p(t, x, y)$ up to positive constants.
\end{abstract}

1. Introduction. Let $(\mathcal{E}, \mathcal{F})$ be a jump type regular Dirichlet form on $L^{2}\left(\boldsymbol{R}^{d}\right)$ as follows:

$$
\mathcal{E}(u, u)=\int_{\boldsymbol{R}^{d} \times \boldsymbol{R}^{d}}(u(y)-u(x))^{2} J(x, y) d x d y, \quad \mathcal{F}={\overline{\left\{C_{c}\left(\boldsymbol{R}^{d}\right)\right.}}_{\mathcal{E}_{1}^{1 / 2}},
$$

where $C_{c}\left(\boldsymbol{R}^{d}\right)$ is the family of all continuous functions on $\boldsymbol{R}^{d}$ with compact supports, $\mathcal{E}_{1}(u, u)=\mathcal{E}(u, u)+\int_{\boldsymbol{R}^{d}} u^{2}(x) d x$ and $J(x, y)$ is a symmetric Borel function satisfying

$$
\frac{\kappa_{1}}{|x-y|^{d} \phi(|x-y|)} \leq J(x, y) \leq \frac{\kappa_{2}}{|x-y|^{d} \phi(|x-y|)}
$$

for positive constants $\kappa_{1}, \kappa_{2}$ and a positive strictly increasing function $\phi$. In the sequel, we consider the case when $\phi$ is written by

$$
\phi(r)=r^{\alpha} \exp (m(r-1) \vee 0), \quad 0<\alpha<2, \quad m \geq 0 .
$$

By the general theory of regular Dirichlet forms, there exists a unique Hunt process associated with $(\mathcal{E}, \mathcal{F})$ and denote it by $\left\{X_{t}\right\}_{t \geq 0}$ (Chapter 7 of [9]). We call the associated process $\left\{X_{t}\right\}_{t \geq 0}$ $\alpha$-stable-like (relativistic $\alpha$-stable-like) if $m=0(m>0)$. Let $\left\{P_{t}\right\}_{t \geq 0}$ be the semigroup generated by the Hunt process $\left\{X_{t}\right\}_{t \geq 0}$ :

$$
P_{t} f(x)=\boldsymbol{E}_{x}\left[f\left(X_{t}\right)\right]=\int_{\boldsymbol{R}^{d}} p(t, x, y) f(y) d y,
$$

where $p(t, x, y)$ is the transition probability density of $\left\{X_{t}\right\}_{t \geq 0}$.

Let $\mu$ be a positive, Green-tight, smooth Radon measure on $\boldsymbol{R}^{d}$ (for the definition, see Definition 3.2). We consider the Schrödinger form

$$
\mathcal{E}^{\mu}(u, u):=\mathcal{E}(u, u)-\int_{\boldsymbol{R}^{d}} u^{2} d \mu .
$$

2010 Mathematics Subject Classification. Primary 60J45; Secondary 60J75, 35J10, 60J35, 31C25.

Key words and phrases. Dirichlet forms, perturbation, heat kernel, Markov processes. 
Denoting the corresponding semigroup by $\left\{P_{t}^{\mu}\right\}_{t \geq 0}$, we know that $\left\{P_{t}^{\mu}\right\}_{t \geq 0}$ also admits an integral kernel $p^{\mu}(t, x, y)$ on $(0, \infty) \times \boldsymbol{R}^{d} \times \boldsymbol{R}^{d}$. The purpose of this paper is to establish a necessary and sufficient condition on $\mu$ for $p^{\mu}(t, x, y)$ to have the same two-sided estimates as $p(t, x, y)$ up to positive constants. We call this phenomenon the stability of fundamental solution. Takeda [15] deals with the stability of fundamental solutions when the underlying process $\left\{X_{t}\right\}_{t \geq 0}$ is a transient Brownian motion. More precisely, assume $\mu$ is in a certain class. Then it is shown in [15] that the stability of fundamental solution holds if and only if

$$
\lambda(\mu):=\inf \left\{\frac{1}{2} \boldsymbol{D}(u, u) ; u \in H^{1}\left(\boldsymbol{R}^{d}\right), \int_{\boldsymbol{R}^{d}} u^{2} d \mu=1\right\}>1,
$$

where $\boldsymbol{D}$ is the Dirichlet integral and $H^{1}\left(\boldsymbol{R}^{d}\right)$ is the 1-order Sobolev space. Note that the formula (5) describes the smallness of the measure $\mu$. Indeed, if $\mu_{1} \leq \mu_{2}$, then $\lambda\left(\mu_{1}\right) \geq \lambda\left(\mu_{2}\right)$. We assume that $(\mathcal{E}, \mathcal{F})$ is transient. Our objective is to extend the result to the symmetric jump process generated by $(\mathcal{E}, \mathcal{F})$, and our main theorem is as follows:

THEOREM 1.1. Let $(\mathcal{E}, \mathcal{F})$ be a transient Dirichlet form satisfying (1)-(3). Suppose $\mu$ is a Green-tight measure of 0-order finite energy integral (for the definition, see Definition 3.2 and (29)). Then the stability of fundamental solution is valid if and only if $\mu$ satisfies

$$
\inf \left\{\mathcal{E}(u, u) ; u \in \mathcal{F}, \int_{\boldsymbol{R}^{d}} u^{2} d \mu=1\right\}>1
$$

This paper is organized as follows: in the next section we review two properties for jump processes in our framework: one is the conservativeness of processes given by [11], and the other is the two-sided heat kernel estimates given by [5, 7]. We also give the two-sided estimates for the Green kernel. In Section 3 we will give the definition of some classes of smooth measures i.e. Kato class $\mathcal{K}$, the Green-tight Kato class $\mathcal{K}_{\infty}$ and so on. In Section 4 we prove the main result following the arguments of $[8,14] . C_{i}$ 's are unimportant positive constants varying from line to line.

The author would like to thank Professor Masayoshi Takeda for helpful suggestions and comments.

2. Preliminaries. Let $\left\{X_{t}\right\}_{t \geq 0}$ be the Hunt process associated with the Dirichlet form $(\mathcal{E}, \mathcal{F})$ defined by (1)-(3). First we review the conservativeness of $\left\{X_{t}\right\}_{t \geq 0}$.

Proposition 2.1. The Hunt process $\left\{X_{t}\right\}_{t \geq 0}$ is conservative, that is, the lifetime of $\left\{X_{t}\right\}_{t \geq 0}$ is equal to $\infty$.

PROOF. We can easily see that $J(x, y)$ is a symmetric function satisfying

$$
M:=\sup _{x \in \boldsymbol{R}^{d}} \int_{\boldsymbol{R}^{d} \backslash\{x\}}\left(1 \wedge|x-y|^{2}\right) J(x, y) d y<\infty .
$$

Then we can show the conservativeness by the same argument as in [11].

Next we review the two-sided heat kernel estimates given by Chen and Kumagai et al. 
THEOREM 2.2. Let $\left\{X_{t}\right\}_{t \geq 0}$ be the Hunt process generated by a Dirichlet form satisfying (1)-(3).

(i) Suppose $m=0$ in (3). Then there exist positive constants $C_{1}$ and $C_{2}$ such that for $t>0$ and $x, y \in \boldsymbol{R}^{d}$

$$
\begin{gathered}
C_{1}\left(\frac{1}{\left(\phi^{-1}(t)\right)^{d}} \wedge \frac{t}{|x-y|^{d} \phi(|x-y|)}\right) \leq p(t, x, y) \\
\quad \leq C_{2}\left(\frac{1}{\left(\phi^{-1}(t)\right)^{d}} \wedge \frac{t}{|x-y|^{d} \phi(|x-y|)}\right),
\end{gathered}
$$

where $\phi^{-1}$ is the inverse function of $\phi$.

(ii) Suppose $m>0$ in (3). Then there exist positive constants $C_{i}(i=1, \ldots, 4)$ such that for $0<t \leq 1$ and $x, y \in \boldsymbol{R}^{d}$

$$
\begin{gathered}
C_{1}\left(\frac{1}{\left(\phi^{-1}(t)\right)^{d}} \wedge \frac{t}{|x-y|^{d} \phi\left(C_{2}|x-y|\right)}\right) \leq p(t, x, y) \\
\leq C_{3}\left(\frac{1}{\left(\phi^{-1}(t)\right)^{d}} \wedge \frac{t}{|x-y|^{d} \phi\left(C_{4}|x-y|\right)}\right) .
\end{gathered}
$$

(iii) Suppose $m>0$ in (3). Then there exist positive constants $C_{i}(i=1, \ldots, 8)$ such that for $t \geq 1$ and $x, y \in \boldsymbol{R}^{d}$

$$
\begin{aligned}
& C_{1} t^{-\frac{d}{2}} \exp \left(-\frac{C_{2}|x-y|^{2}}{t}\right) \leq p(t, x, y) \\
& \quad \leq C_{3} t^{-\frac{d}{2}} \exp \left(-\frac{C_{4}|x-y|^{2}}{t}\right) \quad(1 \vee|x-y| \leq t),
\end{aligned}
$$

and

$$
\begin{aligned}
C_{5} t^{-\frac{d}{2}} \exp \left(-C_{6}|x-y|\right) & \leq p(t, x, y) \\
& \leq C_{7} t^{-\frac{d}{2}} \exp \left(-C_{8}|x-y|\right) \quad(1 \leq t \leq|x-y|)
\end{aligned}
$$

In order to obtain the two-sided estimates for the Green kernel of $\left\{X_{t}\right\}_{t \geq 0}$, let us introduce the function space $\mathcal{G}$ as follows:

$$
\mathcal{G}=\left\{g ; g \text { is a positive decreasing function on }(0, \infty), \exists C_{1}, C_{2} \text { s.t. } C_{1} \leq \frac{g(2 r)}{g(r)} \leq C_{2}\right\},
$$

where $C_{1}$ and $C_{2}$ are positive constants not depending on $r>0$.

Proposition 2.3. Let $\left\{X_{t}\right\}_{t \geq 0}$ be the Hunt process generated by a Dirichlet form $(\mathcal{E}, \mathcal{F})$ satisfying (1)-(3). Then there exist positive constants $C_{3}, C_{4}$ and $g \in \mathcal{G}$ such that for all $x, y \in \boldsymbol{R}^{d}$ with $x \neq y$,

$$
C_{3} g(|x-y|) \leq G(x, y) \leq C_{4} g(|x-y|) .
$$


PROOF. First we consider the case $m=0$. Since $\phi(r)=r^{\alpha}$, (7) can be rewritten as

$$
C_{1}\left(t^{-\frac{d}{\alpha}} \wedge \frac{t}{|x-y|^{d+\alpha}}\right) \leq p(t, x, y) \leq C_{2}\left(t^{-\frac{d}{\alpha}} \wedge \frac{t}{|x-y|^{d+\alpha}}\right) .
$$

Noting that the process is transient by $\alpha<d$, we can obtain

$$
\begin{aligned}
\int_{0}^{\infty}\left(t^{-\frac{d}{\alpha}} \wedge \frac{t}{|x-y|^{d+\alpha}}\right) d t & =\int_{0}^{|x-y|^{\alpha}} \frac{t}{|x-y|^{d+\alpha}} d t+\int_{|x-y|^{\alpha}}^{\infty} t^{-\frac{d}{\alpha}} d t \\
& =\frac{1}{2|x-y|^{d-\alpha}}+\left(\frac{d}{\alpha}-1\right)^{-1} \frac{1}{|x-y|^{d-\alpha}}=\frac{C_{3}}{|x-y|^{d-\alpha}} .
\end{aligned}
$$

Therefore, by taking $g(r)=r^{\alpha-d}$, the assertion follows.

Next we consider the case $m \neq 0$. The transience of the process implies $d \geq 3$. We have (11) for $0 \leq t \leq 1$ and $|x-y| \leq 1$ if we change positive constants appropriately. Thus combining this with (9), we can obtain the upper bound:

$$
\begin{aligned}
\int_{0}^{\infty} p(t, x, y) d t= & \int_{0}^{1} p(t, x, y) d t+\int_{1}^{\infty} p(t, x, y) d t \\
\leq & \int_{0}^{1} C_{1}\left(t^{-\frac{d}{\alpha}} \wedge \frac{t}{|x-y|^{d+\alpha}}\right) d t \\
& +\int_{1}^{\infty} C_{2} t^{-\frac{d}{2}} \exp \left(-\frac{C_{3}|x-y|^{2}}{t}\right) d t \\
\leq & C_{4}\left(\int_{0}^{|x-y|^{\alpha}} \frac{t}{|x-y|^{d+\alpha}} d t+\int_{|x-y|^{\alpha}}^{\infty} t^{-\frac{d}{\alpha}} d t+\int_{|x-y|^{2}}^{\infty} t^{-\frac{d}{2}} d t\right) \\
\leq & C_{5}\left(\frac{1}{|x-y|^{d-\alpha}}+\frac{1}{|x-y|^{d-2}}\right) \leq \frac{C_{6}}{|x-y|^{d-\alpha}} .
\end{aligned}
$$

For the lower bound, we have

$$
\int_{0}^{\infty} p(t, x, y) d t \geq \int_{0}^{|x-y|^{\alpha}} C_{1} \frac{t}{|x-y|^{d+\alpha}} d t=\frac{C_{2}}{|x-y|^{d-\alpha}} .
$$

Combining (12) and (13), we have for $|x-y| \leq 1$

$$
\frac{C_{1}}{|x-y|^{d-\alpha}} \leq G(x, y) \leq \frac{C_{2}}{|x-y|^{d-\alpha}} .
$$

When $|x-y| \geq 1$ and $0 \leq t \leq 1$, it follows from (8) that

$$
C_{1} t \exp \left(-C_{2}|x-y|\right) \leq p(t, x, y) \leq C_{3} t \exp \left(-C_{4}|x-y|\right) .
$$


Thus combining this with (9)-(10), we obtain the upper bound:

$$
\begin{aligned}
\int_{0}^{\infty} p(t, x, y) d t \leq & \int_{0}^{1} C_{3} t \exp \left(-C_{4}|x-y|\right) d t \\
& +\int_{1}^{|x-y|} C_{5} t^{-\frac{d}{2}} \exp \left(-C_{6}|x-y|\right) d t \\
& +\int_{|x-y|}^{\infty} C_{7} t^{-\frac{d}{2}} \exp \left(-\frac{C_{8}|x-y|^{2}}{t}\right) d t \\
\leq & C_{9} \exp \left(-C_{10}|x-y|\right)+\int_{0}^{|x-y|} C_{7}|x-y|^{2-d_{S}} s^{\frac{d}{2}-2} \exp \left(-C_{8} s\right) d s \\
\leq & \frac{C_{11}}{|x-y|^{d-2}} .
\end{aligned}
$$

We can also obtain the lower bound:

$$
\begin{aligned}
\int_{0}^{\infty} p(t, x, y) d t & \geq \int_{|x-y|}^{\infty} C_{12} t^{-\frac{d}{2}} \exp \left(-\frac{C_{13}|x-y|^{2}}{t}\right) d t \\
& \geq \int_{0}^{1} C_{12}|x-y|^{2-d} s^{\frac{d}{2}-2} \exp \left(-C_{13} s\right) d s \geq \frac{C_{14}}{|x-y|^{d-2}} .
\end{aligned}
$$

We thus have for $|x-y| \geq 1$

$$
\frac{C_{1}}{|x-y|^{d-2}} \leq G(x, y) \leq \frac{C_{2}}{|x-y|^{d-2}}
$$

by (15) and (16). On account of (14) and (17), we have the proposition for $g(r)=r^{2-d} \vee r^{\alpha-d}$.

3. Properties of Kato class measures. We first review the notions of capacity and some classes of measures based on [9]. Denote by $\mathcal{O}$ the family of all open subset of $\boldsymbol{R}^{d}$. For $A \in \mathcal{O}$, we define

$$
\mathcal{L}_{A}=\{u \in \mathcal{F} ; u \geq 1 \text { a.e. on } A\}, \quad \operatorname{Cap}(A)= \begin{cases}\inf _{u \in \mathcal{L}_{A}} \mathcal{E}_{1}(u, u), & \mathcal{L}_{A} \neq \emptyset \\ \infty, & \mathcal{L}_{A}=\emptyset\end{cases}
$$

and for any set $A \subset \boldsymbol{R}^{d}$, we let

$$
\operatorname{Cap}(A)=\inf _{B \in \mathcal{O}, A \subset B} \operatorname{Cap}(B) .
$$

We call this capacity of $A$.

A positive Radon measure $\mu$ on $\boldsymbol{R}^{d}$ is said to be of finite energy integral if

$$
\int_{\boldsymbol{R}^{d}}|v(x)| \mu(d x) \leq C \mathcal{E}_{1}(v, v) \quad\left(v \in \mathcal{F} \cap C_{c}\left(\boldsymbol{R}^{d}\right)\right)
$$

for some positive constant $C$. We denote by $\mathcal{S}_{0}$ the family of positive Radon measures of finite energy integral. Moreover, we define a subset $\mathcal{S}_{00}$ of $\mathcal{S}_{0}$ by

$$
\mathcal{S}_{00}=\left\{\mu \in \mathcal{S}_{0} ; \mu\left(\boldsymbol{R}^{d}\right)<\infty,\left\|U_{1} \mu\right\|_{\infty}<\infty\right\},
$$


where $U_{1} \mu$ is the element of $\mathcal{F}$ satisfying $\mathcal{E}_{1}\left(U_{1} \mu, v\right)=\int_{\boldsymbol{R}^{d}} v d \mu$ and $\|\cdot\|_{\infty}$ stands for the norm of $L^{\infty}\left(\boldsymbol{R}^{d}\right)$.

Using these notions, we define smooth measures and smooth measures in the strict sense. A positive Borel measure $\mu$ is said to be smooth if it satisfies the following two conditions:

(i) $\mu$ charges no set of zero capacity,

(ii) there exists an increasing sequence $\left\{F_{n}\right\}$ of closed sets such that $\mu\left(F_{n}\right)<\infty$ for all $n \in N$ and $\lim _{n \rightarrow \infty} \operatorname{Cap}\left(K \backslash F_{n}\right)=0$ for any compact set $K$.

Furthermore, a positive Borel measure $\mu$ is said to be smooth in the strict sense if there exists a sequence $\left\{E_{n}\right\}$ of Borel sets increasing to $\boldsymbol{R}^{d}$ such that $1_{E_{n}} \cdot \mu \in \mathcal{S}_{00}$ for each $n$ and $\boldsymbol{P}_{x}\left(\lim _{n \rightarrow \infty} T_{\boldsymbol{R}^{d} \backslash E_{n}}=\infty\right)=1$ for any $x \in \boldsymbol{R}^{d}$, where $T_{\boldsymbol{R}^{d} \backslash E_{n}}:=\inf \left\{t>0 ; X_{t} \in \boldsymbol{R}^{d} \backslash E_{n}\right\}$. We denote by $\mathcal{S}_{1}$ the totality of the smooth measures in the strict sense. In the sequel, we assume that $\mu$ is smooth in the strict sense and define some subclasses of $\mathcal{S}_{1}$.

Definition 3.1. A smooth measure in the strict sense $\mu$ is said to be in Kato class ( $\mu \in \mathcal{K}$ in notation), if it holds that

$$
\lim _{\beta \rightarrow \infty} \sup _{x \in \boldsymbol{R}^{d}} \int_{\boldsymbol{R}^{d}} G_{\beta}(x, y) \mu(d y)=0,
$$

where $G_{\beta}(x, y)=\int_{0}^{\infty} e^{-\beta t} p(t, x, y) d t$.

The following definition on the Green-tight smooth measures of Kato class is taken from Takeda [13].

Definition 3.2. A measure $\mu \in \mathcal{K}$ is said to be Green-tight ( $\mu \in \mathcal{K}_{\infty}$ in notation), if for any $\varepsilon>0$, there exist a compact set $K_{\varepsilon}$ and a positive constant $\delta_{\varepsilon}$ such that

$$
\sup _{x \in \boldsymbol{R}^{d}} \int_{K_{\varepsilon}^{c}} G(x, y) \mu(d y) \leq \varepsilon
$$

and for any $B \subset K_{\varepsilon}$ with $\mu(B)<\delta_{\varepsilon}$, it holds that

$$
\sup _{x \in \boldsymbol{R}^{d}} \int_{B} G(x, y) \mu(d y) \leq \varepsilon .
$$

The following definition on the conditionally Green-tight smooth measures of Kato class is also taken from Takeda [13].

Definition 3.3. A measure $\mu \in \mathcal{K}$ is said to be in $\mathcal{S}_{\infty}$, if for any $\varepsilon>0$, there exists a compact set $K_{\varepsilon}$ and a positive constant $\delta_{\varepsilon}$ such that

$$
\sup _{x, z \in \boldsymbol{R}^{d}} \int_{K_{\varepsilon}^{c}} \frac{G(x, y) G(y, z)}{G(x, z)} \mu(d y) \leq \varepsilon
$$

and for any $B \subset K_{\varepsilon}$ with $\mu(B)<\delta_{\varepsilon}$, it holds that

$$
\sup _{x, z \in \boldsymbol{R}^{d}} \int_{B} \frac{G(x, y) G(y, z)}{G(x, z)} \mu(d y) \leq \varepsilon .
$$


Let $A_{t}^{\mu}$ be the positive continuous additive functional in the strict sense which is in the Revuz correspondence with $\mu \in \mathcal{S}_{1}$ (Theorem 5.1.7 of [9]): for all positive bounded Borel measurable function $f$ and $\gamma$-excessive function $h$,

$$
\int_{\boldsymbol{R}^{d}} f(x) h(x) \mu(d x)=\lim _{t \rightarrow 0} \frac{1}{t} \boldsymbol{E}_{h d x}\left[\int_{0}^{t} f\left(X_{s}\right) d A_{s}^{\mu}\right] .
$$

There are different definitions for $\mathcal{K}, \mathcal{K}_{\infty}$ and $\mathcal{S}_{\infty}$ in $[3,16]$. We first make sure that these definitions are equivalent each other.

PROPOSITION 3.4. The following assertions are equivalent each other.

(i) $\mu \in \mathcal{K}$;

(ii) $\lim _{t \rightarrow 0} \sup _{x \in \boldsymbol{R}^{d}} \boldsymbol{E}_{x}\left[A_{t}^{\mu}\right]=0$;

(iii) $\lim _{a \rightarrow 0} \sup _{x \in \boldsymbol{R}^{d}} \int_{|x-y| \leq a} G(x, y) \mu(d y)=0$.

ProOF. Note that

$$
\boldsymbol{E}_{x}\left[A_{t}^{\mu}\right]=\int_{\boldsymbol{R}^{d}} \int_{0}^{t} p(s, x, y) d s \mu(d y)
$$

by [1]. We see from Lemma 3.1 of [10] that the right hand side of (21) tends to 0 as $t \rightarrow 0$, if and only if (18) holds, which implies the equivalence between (i) and (ii).

As for the equivalence between (ii) and (iii), Kuwae and Takahashi proved in Theorem 3.2 of [10] for more general Markov processes, but we give another proof here by checking some conditions in [17]. Let

$$
\begin{aligned}
\tau_{B(x, r)} & :=\inf \left\{t>0 ; X_{t} \notin B(x, r)\right\}, \\
T_{B(x, r)} & :=\inf \left\{t>0 ; X_{t} \in B(x, r)\right\} .
\end{aligned}
$$

On account of Theorem 1 of [17], it is sufficient to prove the following three formulae:

$$
\begin{aligned}
\alpha_{0} & :=\sup _{t>0} \inf _{r>0} \sup _{x \in \boldsymbol{R}^{d}} \boldsymbol{P}_{x}\left(\tau_{B(x, r)}>t\right)<1, \\
\beta_{0} & :=\sup _{r>0} \inf _{t>0} \sup _{x \in \boldsymbol{R}^{d}} \boldsymbol{P}_{x}\left(\tau_{B(x, r)}<t\right)<1, \\
\lambda_{0} & :=\sup _{u>0} \inf _{r>0} \sup _{|x-y| \geq u} \boldsymbol{P}_{y}\left(T_{B(x, r)}<\infty\right)<1 .
\end{aligned}
$$

We first show that there exists a positive constant $C_{5}$ such that for all $x \in \boldsymbol{R}^{d}$ and $0<r<1 / 4$,

$$
\boldsymbol{E}_{x}\left[\tau_{B(x, r)}\right] \leq C_{5} \phi(r) .
$$

Indeed, using the Lèvy system formula, we have

$$
\begin{aligned}
1 & \geq \boldsymbol{P}_{x}\left(X_{\tau_{B(x, r)}} \notin \overline{B(x, 2 r)}\right)=\boldsymbol{E}_{x}\left[\int_{0}^{\tau_{B(x, r)}} \int_{\overline{B(x, 2 r)}^{c}} J\left(X_{s}, u\right) d u d s\right] \\
& \geq \boldsymbol{E}_{x}\left[\int_{0}^{\tau_{B(x, r)}} \int_{\frac{\kappa_{1}}{B(x, 2 r)}} \frac{}{\left|X_{s}-u\right|^{d} \phi\left(\left|X_{s}-u\right|\right)} d u d s\right] \\
& \geq C_{6} \int_{3 r}^{\infty} \frac{1}{\rho \phi(\rho)} d \rho \boldsymbol{E}_{x}\left[\tau_{B(x, r)}\right] \geq C_{6} \int_{3 r}^{1} \rho^{-\alpha-1} d \rho \boldsymbol{E}_{x}\left[\tau_{B(x, r)}\right]
\end{aligned}
$$




$$
\geq C_{7} r^{-\alpha} \boldsymbol{E}_{x}\left[\tau_{B(x, r)}\right]=\frac{C_{7}}{\phi(r)} \boldsymbol{E}_{x}\left[\tau_{B(x, r)}\right] .
$$

This is an extension of Theorem 5.1 in [5]. Therefore we see that

$$
\boldsymbol{P}_{x}\left(\tau_{B(x, r)}>t\right) \leq \frac{C_{8} \phi(r)}{t}
$$

and obtain (22) with $\alpha_{0}=0$.

Applying Proposition 4.9 of Chen and Kumagai [6], we see that for arbitrary $\varepsilon>0$ there exists $\gamma_{\varepsilon}>0$ such that for $0<r<1$ and $x \in \boldsymbol{R}^{d}$

$$
\boldsymbol{P}_{x}\left(\tau_{B(x, r)}<\gamma_{\varepsilon} \phi(r)\right) \leq \varepsilon
$$

and that for fixed $r>0$,

$$
\inf _{t>0} \sup _{x \in \boldsymbol{R}^{d}} \boldsymbol{P}_{x}\left(\tau_{B(x, r)}<t\right) \leq \sup _{x \in \boldsymbol{R}^{d}} \boldsymbol{P}_{x}\left(\tau_{B(x, r)}<\gamma_{\varepsilon}\left(r^{\alpha} \wedge 1\right)\right) \leq \varepsilon .
$$

Hence, we obtain (23) with $\beta_{0}=0$.

We can prove (24) in the same way as in Lemma 5 of [17], which deals with Lévy processes. Note that there exist positive constants $C_{1}, C_{2}$ and $g \in \mathcal{G}$ such that

$$
C_{1} g(|x-y|) \leq G(x, y) \leq C_{2} g(|x-y|) .
$$

Fix $u>0$ and $b \geq 1$. Noting that $|y-z| \geq 2 b r$ if $|x-y| \geq u, 0<r \leq \frac{u}{2 b+1}$ and $|z-x| \leq r$, we have

$$
\begin{aligned}
& C_{2} g(2 b r) r^{d} \geq \int_{B(x, r)} G(y, z) d z \geq \boldsymbol{E}_{y}\left[\int_{T_{B(x, r)}}^{\infty} 1_{B(x, r)}\left(X_{t}\right) d t\right] \\
& =\boldsymbol{E}_{y}\left[\boldsymbol{E}_{X_{T_{B(x, r)}}}\left[\int_{0}^{\infty} 1_{B(x, r)}\left(X_{t}\right) d t\right]: T_{B(x, r)}<\infty\right] \\
& \geq \boldsymbol{P}_{y}\left(T_{B(x, r)}<\infty\right) \cdot \inf _{|w-x| \leq r} \int_{B(x, r)} G(w, z) d z \\
& \geq \boldsymbol{P}_{y}\left(T_{B(x, r)}<\infty\right) \cdot \inf _{|w-x| \leq r} \int_{B(x, r)} C_{1} g(|w-z|) d z \\
& \geq C_{1} g(2 r) r^{d} \boldsymbol{P}_{y}\left(T_{B(x, r)}<\infty\right) \text {. }
\end{aligned}
$$

We thus see that

$$
\inf _{r>0} \sup _{|x-y| \geq u} \boldsymbol{P}_{y}\left(T_{B(x, r)}<\infty\right) \leq \varlimsup_{r \rightarrow 0} \frac{C_{2} g(2 b r)}{C_{1} g(2 r)} .
$$

For a sufficiently large $b$, the right hand side of the above formula is smaller than 1 . Hence, we obtain (24).

In the sequel, we assume $\mu \in \mathcal{K}$. The following proposition says that the two notions on the Green-tight smooth measures of Kato class from [3] and [13] coincide with each other.

PROPOSITION 3.5. For $\mu \in \mathcal{K}$, the following assertions are equivalent each other.

(i) $\mu \in \mathcal{K}_{\infty}$. 
(ii) For any $\varepsilon>0$ there exist a set $F_{\varepsilon}$ of $\mu$-finite measure and a positive constant $\tilde{\delta}_{\varepsilon}$ such that

$$
\sup _{x \in \boldsymbol{R}^{d}} \int_{F_{\varepsilon}^{c}} G(x, y) \mu(d y) \leq \varepsilon
$$

and for any $B \subset F_{\varepsilon}$ with $\mu(B)<\tilde{\delta}_{\varepsilon}$,

$$
\sup _{x \in \boldsymbol{R}^{d}} \int_{B} G(x, y) \mu(d y) \leq \varepsilon .
$$

(iii) It holds that

$$
\lim _{r \rightarrow \infty} \sup _{x \in \boldsymbol{R}^{d}} \int_{|y|>r} G(x, y) \mu(d y)=0 .
$$

PROOF. Let $K_{\varepsilon}$ and $\delta_{\varepsilon}$ be a compact set and a positive constant in (19)-(20) respectively. Since $\mu \in \mathcal{K}$ and $g(|x-y|) \rightarrow \infty$ as $|x-y| \rightarrow 0$, (iii) of Proposition 3.4 implies that $\mu(B(x, a)) \rightarrow 0$ as $a \rightarrow 0$ uniformly in $x \in \boldsymbol{R}^{d}$. We thus obtain $\mu\left(K_{\varepsilon}\right)<\infty$, which implies (ii) from (i).

We follow the proof of Theorem 2.1 (3) in [3]. Let $F_{\varepsilon}$ and $\tilde{\delta}_{\varepsilon}$ be a set of $\mu$-finite measure and a positive constant satisfying (ii). Since

$$
\mu\left(\overline{B(0, R)}^{c} \cap F_{\varepsilon}\right) \rightarrow 0 \quad(R \rightarrow \infty),
$$

there exists a positive constant $R_{\varepsilon}$ such that

$$
\sup _{x \in \boldsymbol{R}^{d}} \int_{\frac{B\left(0, R_{\varepsilon}\right)}{c} \cap F_{\varepsilon}} G(x, y) \mu(d y) \leq \varepsilon .
$$

We thus obtain

$$
\sup _{x \in \boldsymbol{R}^{d}} \int_{{\overline{B\left(0, R_{\varepsilon}\right)}}^{c}} G(x, y) \mu(d y) \leq \sup _{x \in \boldsymbol{R}^{d}}\left(\int_{F_{\varepsilon}^{c}} G(x, y) \mu(d y)+\int_{{\overline{B\left(0, R_{\varepsilon}\right)}}^{c} \cap F_{\varepsilon}} G(x, y) \mu(d y)\right) \leq 2 \varepsilon .
$$

It holds that for $B \subset \overline{B\left(0, R_{\varepsilon}\right)}$ with $\mu(B)<\tilde{\delta}_{\varepsilon}$

$$
\sup _{x \in \boldsymbol{R}^{d}} \int_{B} G(x, y) \mu(d y) \leq \sup _{x \in \boldsymbol{R}^{d}}\left(\int_{B \cap F_{\varepsilon}} G(x, y) \mu(d y)+\int_{F_{\varepsilon}^{c}} G(x, y) \mu(d y)\right) \leq 2 \varepsilon .
$$

Hence, (i) follows for $K_{\varepsilon}=\overline{B\left(0, R_{\frac{\varepsilon}{2}}\right)}$ and $\delta_{\varepsilon}=\tilde{\delta}_{\frac{\varepsilon}{2}}$.

We easily see that (iii) follows from (i) by choosing the empty set as $B$ and $\overline{B(0, r)}$ as $K$ respectively.

If (iii) is valid, it follows that for arbitrary $\varepsilon>0$ there exists a sufficient large $r_{\varepsilon}>0$ such that

$$
\sup _{x \in \boldsymbol{R}^{d}} \int_{|y|>r_{\varepsilon}} G(x, y) \mu(d y) \leq \frac{\varepsilon}{2} .
$$

Set $K_{\varepsilon}=\overline{B\left(0, r_{\varepsilon}\right)}$. Since $\mu \in \mathcal{K}$, (iii) of Proposition 3.4 implies that there exists a sufficient small positive constant $a_{\varepsilon}$ such that

$$
\sup _{x \in \boldsymbol{R}^{d}} \int_{|x-y| \leq a_{\varepsilon}} G(x, y) \mu(d y) \leq \frac{\varepsilon}{2} .
$$


Thus, it holds that for a measurable set $A \subset K_{\varepsilon}$

$$
\begin{aligned}
\int_{A} G(x, y) \mu(d y) & \leq \int_{B\left(x, a_{\varepsilon}\right)} G(x, y) \mu(d y)+\int_{A \cap B\left(x, a_{\varepsilon}\right)^{c}} G(x, y) \mu(d y) \\
& \leq \frac{\varepsilon}{2}+C_{1} g\left(a_{\varepsilon}\right) \mu(A) .
\end{aligned}
$$

If we choose a sufficient small positive constant $\delta_{\varepsilon}$, the second term of (25) can be smaller than $\varepsilon / 2$ for any set $A$ with $\mu(A)<\delta_{\varepsilon}$.

Next we compare the definition of $\mathcal{S}_{\infty}$ with that in [3]. The following definition on the conditionally Green-tight smooth measures of Kato class is taken from Chen [3].

DEFINITION 3.6. The measure $\mu$ belongs to the class $\hat{\mathcal{S}}_{\infty}$ if for any $\varepsilon>0$ there exist a set $F_{\varepsilon}$ of $\mu$-finite measure and $\tilde{\delta}_{\varepsilon}>0$ such that

$$
\sup _{x, z \in \boldsymbol{R}^{d} \times \boldsymbol{R}^{d} \backslash \Delta} \int_{F_{\varepsilon}^{c}} \frac{G(x, y) G(y, z)}{G(x, z)} \mu(d y) \leq \varepsilon,
$$

$\Delta=\left\{(x, x) ; x \in \boldsymbol{R}^{d}\right\}$ and for any $B \subset F_{\varepsilon}$ with $\mu(B)<\tilde{\delta}_{\varepsilon}$

$$
\sup _{x, z \in \boldsymbol{R}^{d} \times \boldsymbol{R}^{d} \backslash \Delta} \int_{B} \frac{G(x, y) G(y, z)}{G(x, z)} \mu(d y) \leq \varepsilon .
$$

The following proposition can be proved in the same way as (i) $\Rightarrow$ (ii) of Proposition 3.5.

PROPOSITION 3.7. It holds that $\mathcal{S}_{\infty} \subset \hat{\mathcal{S}}_{\infty}$.

The next theorem is proved by means of $3 G$-theorem.

THEOREM 3.8. It holds that $\mathcal{K}_{\infty}=\mathcal{S}_{\infty}=\hat{\mathcal{S}}_{\infty}$.

PRoOF. Note that either $|x-y| \geq|x-z| / 2$ or $|y-z| \geq|x-z| / 2$ holds. If $|x-y| \geq$ $|x-z| / 2$, it follows that

$$
\begin{aligned}
\frac{G(x, y) G(y, z)}{G(x, z)} & \leq \frac{C_{2} g(|x-y|)}{C_{1} g(|x-z|)} G(y, z) \\
& \leq \frac{C_{2} g(|x-z| / 2)}{C_{1} g(|x-z|)} G(y, z) \leq C_{3} G(y, z)=C_{3} G(z, y)
\end{aligned}
$$

by Proposition 2.3. If $|y-z| \geq|x-z| / 2$ we can similarly obtain

$$
\frac{G(x, y) G(y, z)}{G(x, z)} \leq C_{4} G(x, y) .
$$

Combining (26) and (27) we see that

$$
\frac{G(x, y) G(y, z)}{G(x, z)} \leq C_{5}(G(x, y)+G(z, y)),
$$


and thus $\mathcal{K}_{\infty} \subset \mathcal{S}_{\infty}$. Moreover, we see $\hat{\mathcal{S}}_{\infty} \subset \mathcal{K}_{\infty}$ from Corollary 3.1 of [4] and p.4663 of [3]. Combining these results and Proposition 3.7, we obtain $\mathcal{K}_{\infty} \subset \mathcal{S}_{\infty} \subset \hat{\mathcal{S}}_{\infty} \subset \mathcal{K}_{\infty}$ and this is the desired assertion.

By Proposition 2.2 of [3], we can obtain the next corollary:

COROllary 3.9. For $\mu \in \mathcal{K}_{\infty}$,

$$
\sup _{x \in \boldsymbol{R}^{d}} \boldsymbol{E}_{x}\left[A_{\infty}^{\mu}\right]<\infty
$$

4. Proof of Theorem 1.1. In this section we assume $\mu \in \mathcal{K}_{\infty}$ and consider the Schrödinger form $\left(\mathcal{E}^{\mu}, \mathcal{F}\right)$ defined by (4). Denote by $\left\{P_{t}^{\mu}\right\}_{t \geq 0}$ the corresponding semigroup. It is known that $P_{t}^{\mu}$ is written by

$$
P_{t}^{\mu} f(x)=\boldsymbol{E}_{x}\left[\exp \left(A_{t}^{\mu}\right) f\left(X_{t}\right)\right],
$$

where $A_{t}^{\mu}$ is a positive continuous additive functional in the Revuz correspondence with $\mu$. Following [1,2], we see that $\left\{P_{t}^{\mu}\right\}_{t \geq 0}$ admits the integral kernel $p^{\mu}(t, x, y)$ defined on $(0, \infty) \times \boldsymbol{R}^{d} \times \boldsymbol{R}^{d}$.

Noting that $\mathcal{K}_{\infty}=\mathcal{S}_{\infty}$, we can easily prove the following proposition by the same arguments as in Theorem 2.4 and Theorem 3.9 of Takeda [13].

PROPOSITION 4.1. For $\mu \in \mathcal{K}_{\infty}$, the following assertions are equivalent.

(i) $G^{\mu}(x, y):=\int_{0}^{\infty} p^{\mu}(t, x, y) d t<\infty$ for $x \neq y$;

(ii) $\inf \left\{\mathcal{E}(u, u) ; u \in \mathcal{F}, \int_{\boldsymbol{R}^{d}} u^{2} d \mu=1\right\}>1$;

(iii) $\sup _{x \in \boldsymbol{R}^{d}} \boldsymbol{E}_{x}\left[\exp \left(A_{\infty}^{\mu}\right)\right]<\infty$.

Following this proposition, we can prove the 'only if' part of Theorem 1.1. Suppose the stability of fundamental solution holds. Using the Green kernel estimates similarly obtained as in Proposition 2.3, we see that $G^{\mu}(x, y)<\infty$. This is equivalent to (6).

Before proving the 'if' part, we introduce the notion of gaugeability.

Definition 4.2. The Green-tight measure $\mu$ is called gaugeable if $A_{t}^{\mu}$ satisfies (iii) of Proposition 4.1.

Now we prove the 'if' part of Theorem 1.1. The equation (6) and Proposition 4.1 imply that

$$
1 \leq h(x):=\boldsymbol{E}_{x}\left[\exp \left(A_{\infty}^{\mu}\right)\right] \leq C_{1}<\infty
$$

In order to apply Theorem 3.4 in [8], we need to show that there exists $u \in \mathcal{F}_{e}$ such that $h(x)=\exp (u(x))$, where $\mathcal{F}_{e}$ is the extended Dirichlet space, namely the closure of $\mathcal{F}$ with respect to the $\mathcal{E}^{1 / 2}$-norm. Hence we make the following assumption on $\mu$ :

$$
\int_{\boldsymbol{R}^{d} \times \boldsymbol{R}^{d}} G(x, y) \mu(d x) \mu(d y)<\infty .
$$

LEMMA 4.3. For $\mu \in \mathcal{K}_{\infty}$, define $G \mu(x)=\int_{\boldsymbol{R}^{d}} G(x, y) \mu(d y)$. Under assumption (29), $G \mu \in \mathcal{F}_{e}$. 
Proof. This lemma is an extension of Lemma 3.1 in [14]. Let $\mu \in \mathcal{K}_{\infty}$. Then it holds that

$$
\int_{\boldsymbol{R}^{d}} u^{2} d \mu \leq\|G \mu\|_{\infty} \mathcal{E}(u, u)
$$

for $u \in \mathcal{F}_{e}$. For detail, see [12]. Define $\mu_{K}(\cdot)=\mu(K \cap \cdot)$ for a set $K$ of $\mu$-finite measure. Applying (30), we have

$$
\begin{aligned}
\int_{\boldsymbol{R}^{d}} \psi d \mu_{K} & \leq(\mu(K))^{1 / 2}\left(\int_{\boldsymbol{R}^{d}} \psi^{2} d \mu_{K}\right)^{1 / 2} \\
& \leq(\mu(K))^{1 / 2}\left\|G \mu_{K}\right\|_{\infty}^{1 / 2} \mathcal{E}(\psi, \psi)^{1 / 2} .
\end{aligned}
$$

By (28), we see $\left\|G \mu_{K}\right\|_{\infty} \leq\|G \mu\|_{\infty}=\sup _{x \in \boldsymbol{R}^{d}} \boldsymbol{E}_{x}\left[A_{\infty}^{\mu}\right]<\infty$ and consequently $\mu_{K}$ is of finite energy integral in the sense of [9]. We thus have

$$
\begin{aligned}
\int_{\boldsymbol{R}^{d}} \psi d \mu_{K} & \leq \mathcal{E}\left(G \mu_{K}, G \mu_{K}\right)^{1 / 2} \mathcal{E}(\psi, \psi)^{1 / 2} \\
& \leq\left(\int_{\boldsymbol{R}^{d} \times \boldsymbol{R}^{d}} G(x, y) d \mu_{K}(x) d \mu_{K}(y)\right)^{1 / 2} \mathcal{E}(\psi, \psi)^{1 / 2} .
\end{aligned}
$$

We see that $\mu$ is also of finite energy integral and $G \mu \in \mathcal{F}_{e}$ by letting $K$ to $\boldsymbol{R}^{d}$.

Lemma 4.4. Suppose $\mu \in \mathcal{K}_{\infty}$ is gaugeable. Then it holds that

$$
h(x)=1+G(h \mu)(x) .
$$

PROOF. This is an extension of Lemma 3.2 of [14]. Let $\left\{\mathcal{M}_{t}\right\}_{t \geq 0}$ be the filtration equipped with the Hunt process $\left\{X_{t}\right\}_{t \geq 0}$. Set $\bar{M}_{t}=\boldsymbol{E}_{x}\left[\exp \left(A_{\infty}^{\mu}\right) \mid \mathcal{M}_{t}\right]$. By the Markov property, we have

$$
\begin{aligned}
h\left(X_{t}\right) & =\boldsymbol{E}_{X_{t}}\left[\exp \left(A_{\infty}^{\mu}\right)\right]=\boldsymbol{E}_{x}\left[\exp \left(A_{\infty}^{\mu} \circ \theta_{t}\right) \mid \mathcal{M}_{t}\right] \\
& =\exp \left(-A_{t}^{\mu}\right) \boldsymbol{E}_{x}\left[\exp \left(A_{t}^{\mu}+A_{\infty}^{\mu} \circ \theta_{t}\right) \mid \mathcal{M}_{t}\right]=\exp \left(-A_{t}^{\mu}\right) \bar{M}_{t},
\end{aligned}
$$

where $\theta_{t}$ is the shift operator satisfying $X_{t+s}=X_{s} \circ \theta_{t}$ for all $s \geq 0$. Hence, we have

$$
\begin{aligned}
\boldsymbol{E}_{x}\left[\int_{0}^{t} h\left(X_{s}\right) d A_{s}^{\mu}\right] & =\boldsymbol{E}_{x}\left[\int_{0}^{t} \exp \left(-A_{s}^{\mu}\right) \bar{M}_{s} d A_{s}^{\mu}\right] \\
& =\boldsymbol{E}_{x}\left[\bar{M}_{0}\right]-\boldsymbol{E}_{x}\left[\exp \left(-A_{t}^{\mu}\right) \bar{M}_{t}\right]+\boldsymbol{E}_{x}\left[\int_{0}^{t} \exp \left(-A_{s}^{\mu}\right) d \bar{M}_{s}\right] \\
& =h(x)-\boldsymbol{E}_{x}\left[h\left(X_{t}\right)\right],
\end{aligned}
$$

where we use the martingale property of $\left\{\bar{M}_{t}\right\}_{t \geq 0}$. Note that

$$
\lim _{t \rightarrow \infty} h\left(X_{t}\right)=\lim _{t \rightarrow \infty} \exp \left(-A_{t}^{\mu}\right) \bar{M}_{t}=\exp \left(-A_{\infty}^{\mu}\right) \exp \left(A_{\infty}^{\mu}\right)=1 .
$$

We then have the desired result by letting $t \rightarrow \infty$ in (31). 
Since $h$ is a positive bounded function and $G \mu \in \mathcal{F}_{e}, G(h \mu) \in \mathcal{F}_{e}$ and thus $u(x)=$ $\log h(x)=\log (1+G(h \mu)(x)) \in \mathcal{F}_{e}$. Consider Fukushima's decomposition of $G(h \mu)$ :

$$
G(h \mu)\left(X_{t}\right)-G(h \mu)\left(X_{0}\right)=M_{t}^{[G(h \mu)]}+N_{t}^{[G(h \mu)]},
$$

where $M_{t}^{[G(h \mu)]}$ is a martingale additive functional of finite energy and $N_{t}^{[G(h \mu)]}$ is a continuous additive functional of zero energy. Since the left hand side of (32) equals $h\left(X_{t}\right)-h\left(X_{0}\right)$ by Lemma 4.4, $M_{t}^{[G(h \mu)]}$ equals $M_{t}^{[h]}$. Moreover we see from Lemma 5.4.1 of [9] that

$$
N_{t}^{[G(h \mu)]}=-\int_{0}^{t} h\left(X_{s}\right) d A_{s}^{\mu} .
$$

We thus have

$$
h\left(X_{t}\right)-h\left(X_{0}\right)=M_{t}^{[h]}-\int_{0}^{t} h\left(X_{s}\right) d A_{s}^{\mu} .
$$

Now we define a martingale by

$$
M_{t}=\int_{0}^{t} \frac{1}{h\left(X_{s-}\right)} d M_{s}^{[h]}
$$

and denote by $L_{t}$ the unique solution of Doleans-Dade equation: $L_{t}=1+\int_{0}^{t} L_{s-} d M_{s} . L_{t}$ is expressed by

$$
\begin{aligned}
L_{t} & =\exp \left(M_{t}-\frac{1}{2}\left\langle M^{c}\right\rangle_{t}\right) \prod_{0<s \leq t}\left(1+\Delta M_{s}\right) \exp \left(-\Delta M_{S}\right) \\
& =\exp \left(M_{t}-\frac{1}{2}\left\langle M^{c}\right\rangle_{t}\right) \prod_{0<s \leq t} \frac{h\left(X_{S}\right)}{h\left(X_{s-}\right)} \exp \left(1-\frac{h\left(X_{S}\right)}{h\left(X_{s-}\right)}\right),
\end{aligned}
$$

where $M^{c}$ is the continuous part of martingale $M$ and $\left\langle M^{c}\right\rangle$ is the quadratic variation of $M^{c}$. Applying Itô formula to the semimartingale $h\left(X_{t}\right)$ and the function $\log x$, we have

$$
L_{t}=\frac{h\left(X_{t}\right)}{h\left(X_{0}\right)} \exp \left(A_{t}^{\mu}\right) .
$$

We consider the transformed semigroup $\left\{P_{t}^{\mu, h}\right\}_{t \geq 0}$ by $L_{t}$,

$$
P_{t}^{\mu, h} f(x)=\boldsymbol{E}_{x}\left[L_{t} f\left(X_{t}\right)\right]=\frac{1}{h(x)} \boldsymbol{E}_{x}\left[h\left(X_{t}\right) \exp \left(A_{t}^{\mu}\right) f\left(X_{t}\right)\right] .
$$

We then know from Theorem 3.4 of [8] that the Dirichlet form generated by $\left\{P_{t}^{\mu, h}\right\}_{t \geq 0}$ is identified.

Proposition 4.5. There exists a Dirichlet form $\left(\mathcal{E}^{\mu, h}, \mathcal{D}\left(\mathcal{E}^{\mu, h}\right)\right)$ on $L^{2}\left(h^{2} d x\right)$ corresponding to the semigroup $\left\{P_{t}^{\mu, h}\right\}$ and it has the representation as follows:

$$
\begin{gathered}
\mathcal{E}^{\mu, h}(u, v)=\int_{\boldsymbol{R}^{d} \times \boldsymbol{R}^{d}}(u(y)-u(x))(v(y)-v(x)) J(x, y) h(x) h(y) d x d y, \\
\mathcal{D}\left(\mathcal{E}^{\mu, h}\right)=\mathcal{F} .
\end{gathered}
$$


Note that $P_{t}^{\mu, h}$ admits the integral kernel $h(x)^{-1} p^{\mu}(t, x, y) h(y)^{-1}$ with respect to the measure $h^{2}(y) d y$. Since $1 \leq h(x) \leq C_{1}$ for some positive constant $C_{1}$, we see that the form defined by (33) is the regular Dirichlet form on $L^{2}\left(\boldsymbol{R}^{d}\right)$ with jump measure $J_{1}(x, y):=$ $J(x, y) h(x) h(y)$. Moreover, there exists positive constants $\kappa_{3}$ and $\kappa_{4}$ such that

$$
\frac{\kappa_{3}}{|x-y|^{d} \phi(|x-y|)} \leq J_{1}(x, y) \leq \frac{\kappa_{4}}{|x-y|^{d} \phi(|x-y|)} .
$$

Hence, we see that $h(x)^{-1} p^{\mu}(t, x, y) h(y)^{-1}$ has the same two sided estimates as those given in Theorem 2.2 for $p(t, x, y)$. Since $1 \leq h(x) \leq C_{1}$, so does $p^{\mu}(t, x, y)$.

\section{REFERENCES}

[1] S. Albeverio, P. Blanchard And Z.-M. MA, Feynman-Kac semigroups in terms of signed smooth measures, Internat. Ser. Numer. Math. 102 (1991), 1-31.

[2] S. Albeverio And Z.-M. MA, Perturbation of Dirichlet forms -lower semiboundedness, closability, and form cores, J. Funct. Anal. 99 (1991), 332-356.

[ 3 ] Z.-Q. ChEn, Gaugeability and conditional gaugeability, Trans. Amer. Math. Soc. 354 (2002), 4639-4679.

[4] Z.-Q. Chen And R.-M. Song, General gauge and conditional gauge theorems, Ann. Probab. 30 (2002), 1313-1339.

[ 5 ] Z.-Q. Chen And T. Kumagai, Heat kernel estimates for stable-like processes on $d$-sets, Stochastic Process. Appl. 108 (2003), 27-62.

[6] Z.-Q. ChEn AND T. KumAGAi, Heat kernel estimates for jump processes of mixed types on metric measure spaces, Probab. Theory Related Fields 140 (2008), 277-317.

[ 7 ] Z.-Q. Chen, P. Kim And T. Kumagai, Global heat kernel estimates for symmetric jump processes, Trans. Amer. Math. Soc. 363 (2011), 5021-5055.

[ 8 ] Z.-Q. CHEN AND T.-S. ZHANG, Girsanov and Feynman-Kac type transformations for symmetric Markov processes, Ann. Inst. H. Poincaré Probab. Statist. 38 (2002), 475-505.

[9] M. Fukushima, Y. Oshima And M. TAKedA, Dirichlet forms and symmetric Markov processes, Second revised and extended edition, de Gruyter Stud. Math. 19, Walter de Gruyter \& Co., Berlin, 2011.

[10] K. KUWAE AND M. TAKAHASHI, Kato class measures of symmetric Markov processes under heat kernel estimates, J. Funct. Anal. 250 (2007), 86-113.

[11] J. Masamune and T. Uemura, Conservation property of symmetric jump processes, Ann. Inst. Henri Poincaré Probab. Stat. 47 (2011), 650-662.

[12] P. Stollmann And J. Voigt, Perturbation of Dirichlet forms by measures, Potential Anal. 5 (1996), 109138.

[13] M. TAKEDA, Conditional gaugeability and subcriticality of generalized Schrödinger operators, J. Funct. Anal. 191 (2002), 343-376.

[14] M. TAKeDA, Gaugeability for Feynman-Kac functionals with applications to symmetric $\alpha$-stable processes, Proc. Amer. Math. Soc. 134 (2006), 2729-2738.

[15] M. TAKEDA, Gaussian bounds of heat kernels for Schrödinger oeprators on Riemannian manifolds, Bull. Lond. Math. Soc. 39 (2007), 85-94.

[16] M. TAKEDA AND T. UEMURA, Subcriticality and gaugeability for symmetric $\alpha$-stable processes, Forum Math. 16 (2004), 505-517.

[17] Z. ZнаO, A probabilistic principle and generalized Schrödinger perturbation, J. Funct. Anal. 101 (1991), $162-176$. 
Mathematical Institute

TOHOKU UNIVERSITY

SENDAI 980-8578

JAPAN

E-mail address:wada@math.tohoku.ac.jp 